\title{
Particle Image Velocimetry in Molten Plastic
}

\author{
Thomas J. Bress, David R. Dowling \\ Department of Mechanical Engineering, University of Michigan, Ann Arbor, Michigan 48109-2133
}

\begin{abstract}
Injection molding is a standard manufacturing process for plastic parts. The most important process step, mold filling, involves unsteady nonisothermal flow of a non-Newtonian molten plastic. Mold-filling flow largely determines molecular orientation within the final part and thereby influences final part properties. This article describes techniques for successfully applying particle image velocimetry (PIV) to molten plastic flow during injection molding. The primary experimental challenges include the following: engineering optical access to the molten plastic flow at elevated temperatures (230$245^{\circ}$ ) and pressures ( $\left.20 \mathrm{MPa}\right)$, finding particles that survive the thermal-mechanical environment that melts the plastic, and developing experimental and datareduction techniques that allow multiple imperfect planar PIV measurements to be combined. Here, a custom optical-access mold allowed mold center-plane PIV to be performed in molten polystyrene. Simple statistical assessments of the velocimetry data and scaled residuals of the continuity equation suggest that the PIV can be conducted in molten plastics with an uncertainty of $\pm 2 \%$. POLYM. ENG. SCI., 51:730-745, 2011. ๑ 2011 Society of Plastics Engineers
\end{abstract}

\section{INTRODUCTION}

Injection molding is perhaps the most common manufacturing method for forming raw plastic pellets into geometrically complex parts. Mold filling, the critical step in the injection molding process, influences dimensional stability, mechanical properties, residual stresses, warpage, and part-to-part consistency. Mold filling involves unsteady nonisothermal flow of a molten plastic. The experimental considerations and techniques necessary to determine in-mold molten-plastic fluid velocities under industrially relevant injection-molding conditions are presented here.

Generic injection molding is comprised of a few basic steps that are cyclically repeated as individual parts are made. First, room-temperature solid-plastic pellets are sheared, heated, and melted by an auger screw turning

Correspondence to: Thomas Bress; e-mail: tbress@exponent.com Thomas Bress is currently at Exponent Inc., Bowie, MD 20715. Contract grant sponsor: Colonial Engineering Inc. Plastics Provisions. DOI 10.1002/pen.21881

Published online in Wiley Online Library (wileyonlinelibrary.com). (C) 2011 Society of Plastics Engineers within a close-fitting temperature-controlled cylinder called a barrel. This screw also transports the plastic from the mouth of a pellet hopper to the end the cylinder where it collects until it reaches a specified volume (aka a shot) somewhat greater than the volume of the mold cavity. At this point, the screw stops turning and pushes forward as a ram to force the molten plastic shot through a passageway into the mold. When the melted plastic fills the mold, the screw's forward motion slows as melt pressure builds up and the plastic solidifies in the mold. After the plastic has solidified, the mold opens, the part is ejected, and the screw backs up and begins turning again to build up the next volume (or shot) of molten plastic for the next cycle. A more thorough description of injection molding is provided by Dealy and Wissbrun [1].

This study focuses on velocimetry during the moldfilling step where molten plastic enters the mold through a small opening called a gate and fills the mold cavity while forcing air out. Although most mold cavities are much thinner in one dimension than the other two, the resulting flow is three-dimensional (3D) in general and includes a moving plastic-air interface. However, away from this interface or flow front, much of the flow field is well described locally by fully developed unidirectional non-Newtonian viscous flow between parallel or nearly parallel walls. Thus, mid-cavity velocity measurements can potentially be extrapolated to the entire moldcavity volume, and, in a rectangular mold cavity, such mid-cavity measurements can be made using velocimetry techniques based on planar laser-sheet illumination. Therefore, the primary diagnostic in this study is planar digital particle image velocimetry (DPIV) [2]. The primary challenges in applying DPIV to in-mold polymer melt flow involve obtaining optical access at the elevated pressures and temperatures that occur during injection molding, and circumventing the mechanically restricted access that an industrial injection molding machine affords to the flowing plastic. Here, these challenges are overcome through the design, construction, and use of a special optical access mold having a (nearly) rectangular cavity and through the careful selection and use of appropriate high-reflectivity seed particles that survive passage through the barrel of the injection-molding machine. 
Early injection-molding flow-visualization work was largely qualitative [3]. Visualization studies were often performed to examine polymer melt flow fronts [4, 5]. These studies were used to validate theoretical and computational models of injection molding flow $[6,7]$ but did not provide full-field velocity data. More recent (and more quantitative) melt flow visualization studies have been done to study shrinkage [8], melt front propagation [9], the generation of wave-like flow marks [10], and the filling of microscale V-grooves [11]. These more recent studies, however, do not provide the full-field velocity data of polymer melts that is possible with DPIV.

Non-Newtonian velocimetry studies that provide fullfield velocity data have been done. These studies are quantitative but they typically do not involve unsteady flows or molten plastics that solidify, tending to focus instead on steady flow of non-Newtonian fluids in idealized geometries [12].

The study presented here is a direct extension of these prior efforts; it describes the techniques necessary for collection of quantitative in-mold fluid vector-velocity data from the flow of an industrially relevant molten plastic that solidifies in the mold. The techniques described here allow the quantitative study of the in-mold flow that sets the molecular orientation and residual stress fields that lead to plastic-part warpage and anisotropic material properties [1]. In addition, measured in-mold flow field results provide unique validation data for mold filling computations and simulations [13]. Furthermore, such quantitative in-mold velocimetry results may provide new insights into non-Newtonian flows under industrially relevant conditions.

The remainder of this article is divided into four sections. The next section describes the experimental setup including the injection molding machine, the optical access mold, the plastic, the PIV seed particles, and the illumination and imaging systems. This is followed by a discussion of potentially unusual considerations for using PIV in molten plastics during injection molding. The final sections present image analysis techniques and sample results, respectively. A summary of this effort and the conclusions drawn from it are provided in the final section.

\section{EXPERIMENTAL SETUP}

The primary experimental platform was a commercial Cincinnati Milacron VS33 injection- molding machine capable of injecting a $100 \mathrm{cc}$ shot of molten plastic at 125 $\mathrm{MPa}$ into a mold enclosed between its stationary and moving platens. The machine's clamp was capable of holding the platens stationary (and the mold shut) with a force of 30 tons. Its $0.64-\mathrm{m}$-long barrel had three separate heater sections to provide a barrel-axis temperature profile that enabled the turning screw to appropriately melt the plastic pellets provided from a gravity-feed hopper. For the experiments described here, the machine was run in single-shot mode, as opposed to continuous cyclic operation, to accommodate synchronization of the various data collection systems with the mold-filling step. However, the machine and mold were partially warmed up by the production of parts before the collection of quantitative experimental data. The injection-molding machine was run until it produced consistently good specimens, typically 10 or more. Melt-flow velocity data was not collected until this threshold had been reached.

\section{The Optical Access Mold}

The heart of the molten-plastic flow-field measurement scheme was an optical access mold, specially designed for this experiment and described previously [14]. The mold was constructed from a mild tool steel mold base. An isometric exploded view of the mold is shown in Fig. 1.

The mold produced nominally rectangular plaques with dimensions of $109.5 \mathrm{~mm}$ by $66.7 \mathrm{~mm}$ by $6.3 \mathrm{~mm}\left(45 / 16^{\prime \prime}\right.$ by $25 / 8^{\prime \prime}$ by $\left.0.25^{\prime \prime}\right)$. The rectangular shape was chosen to allow planar windows for undistorted viewing and for ease of meshing in computational mold-filling studies. The mold had three windows: one along the largest face (109.5 mm by $66.7 \mathrm{~mm}$ ) for mold center-plane PIV imaging, and two along the small faces at the top and bottom of the cavity $(66.7 \mathrm{~mm}$ by $6.3 \mathrm{~mm})$ to let the particle-illumination laser sheet into and out of the mold.

The portion of the large window in contact with the $230-245^{\circ} \mathrm{C}$ molten plastic was a polished quartz block 19 mm thick. Here, quartz was chosen for its ability to withstand significant temperature gradients without fracturing. A $3 \mathrm{~mm}$ thickness of polycarbonate acted as a buffer between the quartz and three 25-mm-thick pieces of sodalime glass that carried the mold-cavity pressure load. Here, the stiffness of plate glass was required to maintain mold-cavity shape when loaded by the filled-cavity pressure in the liquid plastic. These were followed by another 3-mm-thick layer of polycarbonate, and the whole assembly was held in place by a steel brace and six hardened grade-eight 25.4-mm-diameter bolts. The mold design pressure $(69 \mathrm{MPa})$ and the projected area of the mold cavity $\left(73 \mathrm{~cm}^{2}\right)$ lead to a total large-window design load of $\sim 500 \mathrm{kN}(\sim 50$ metric tons, more that the injectionmolding machine's clamp tonnage).

The main design challenge for this mold was to produce a window that could withstand the high-melt pressure produced during the packing phase. Due to the limited available space beneath the hood of the injection molding machine, three 25.4-mm thick plates of annealed silica glass were chosen to form the main viewing window. Durable clear-plastic plates - made from polycarbonate or acrylic for example-could have been substituted for the plate glass, but the requisite plastic-plate thickness that achieved the same stiffness as the glass would have been difficult to manage and much less transparent. 


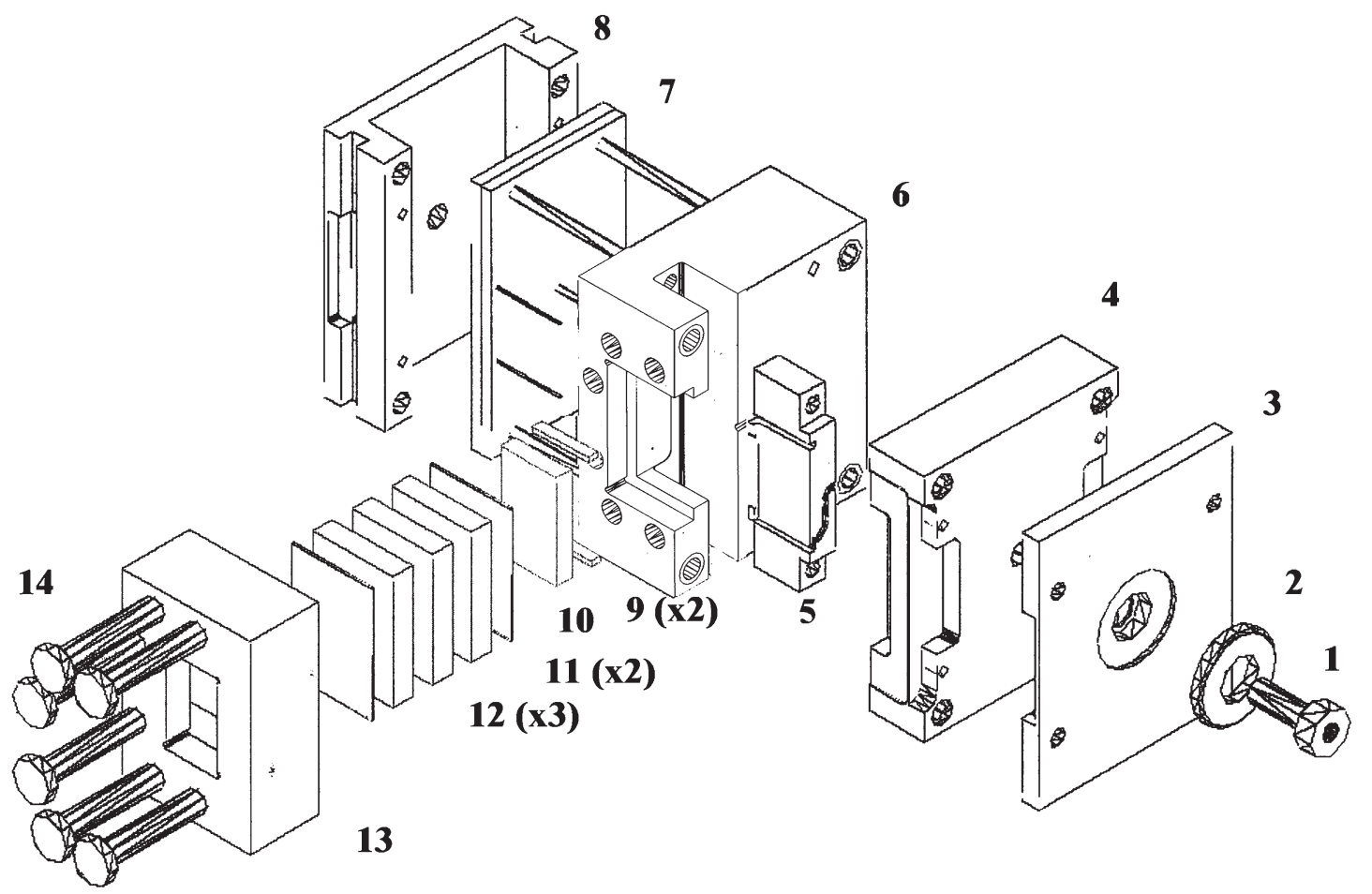

FIG. 1. Exploded view of the optical access mold. 1: Sprue bushing. 2: Guide ring. 3: Top clamping plate. 4: "A" plate. 5: Cavity block. 6: "B" plate. 7: Front and rear ejector plates with ejector pins and return pins. 8: Rear clamping plate. 9: Acrylic entrance and exit windows. 10: 19-mm thick quartz window. 11: 3-mm thick polycarbonate shields. 12: Three 25.4-mm thick glass plates. 13: Steel window brace. 14: Six 25.4-mm diameter hardened steel bolts.

An estimate of the maximum safe melt pressure can be made using standard plate stress formulas [15]. The modulus of rupture for glass is $50 \mathrm{MPa}$ [16] but the recommended range of tensile stresses for annealed glass lies between 4 and $10 \mathrm{MPa}$ [17]. To be conservative, a maximum window tensile stress of $5 \mathrm{MPa}$ was chosen, yielding a safety factor of 10 .

For a rectangular plate under uniform loading, the maximum tensile stress is given by the following:

$$
\sigma_{\max }=\frac{\beta P w^{2}}{t^{2}}
$$

where $P$ is the melt pressure, $w$ is the width of the mold cavity $(66.7 \mathrm{~mm}), t$ is the thickness of the window $(76.2$ $\mathrm{mm}$ ), and $\beta$ is a constant dependent on the boundary conditions and on the aspect ratio of the window [15]. For a rectangular plate with an aspect ratio of 1.6 and with all edges fixed, $\beta=0.2286$. Setting the maximum stress equal to the modulus of rupture of glass and applying a safety factor of 10 gives an estimated maximum melt pressure of $28.5 \mathrm{MPa}$. This estimate was quite accurate. In actual practice, the melt pressure was taken up to 27.6 $\mathrm{MPa}$. Glass breakage was frequent at higher melt pressures. The injection-molding machine used in this work was capable of producing a $69 \mathrm{MPa}$ packing pressure, so the window design was capable of withstanding $40 \%$ of the maximum available packing pressure.
The smaller top and bottom mold windows were only marginally less problematic. These were rectangular blocks of either quartz or acrylic and were held in place in slots machined into a steel block (part no. 5 in Fig. 1) inserted into the mold base.

A schematic of the mold cavity, windows, and cavity orientation with respect to the mold's parting plane is shown in Fig. 2. There is $1 / 2^{\circ}$ of draft along the top and bottom windowed faces as well as on the steel face opposite the large window to facilitate ejection of the molded

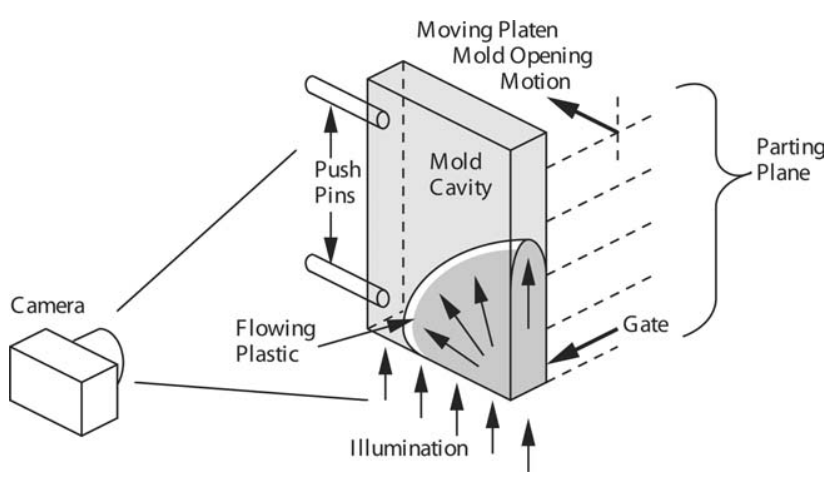

FIG. 2. Melt flow visualization schematic. The liquid plastic enters the rectangular mold at the lower right corner. The mold cavity is contained within the portion of the mold base attached to the moving platen. The flowing plastic is viewed through a window over the planform area of the part. Light enters and exits the mold through the windows on its top and bottom. 


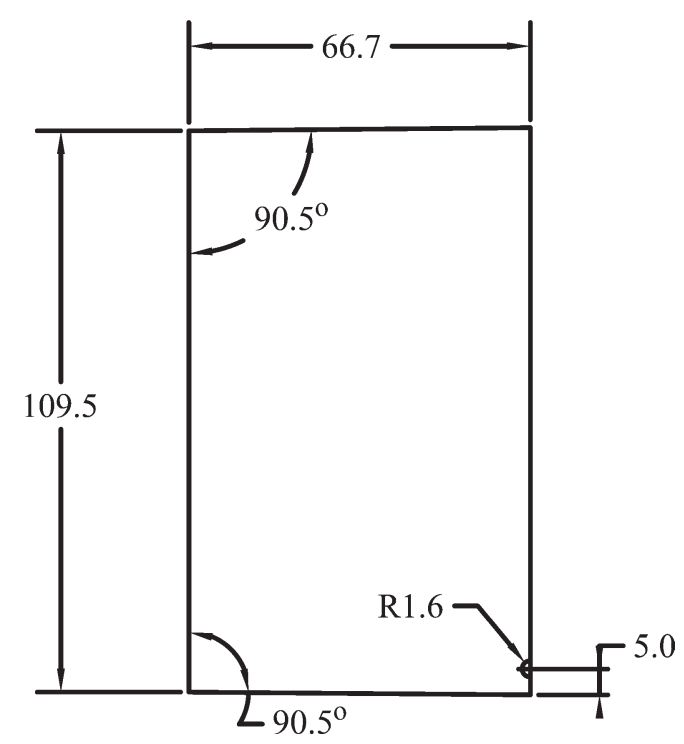

FIG. 3. Schematic of the mold cavity. The top and bottom edges include $1 / 2^{\circ}$ of draft to aid in the ejection of the part from the mold. The gate is $5 \mathrm{~mm}$ above the lower right corner of the mold. The mold cavity is $6 \mathrm{~mm}$ thick. All dimensions are in millimeters.

parts. The nontransparent interior surfaces of the mold were steam treated to provide a uniform blue-black finish to improve the contrast of the PIV images.

Typically, flat plaques such as those made in this study would be made with one of the large $(109.5 \mathrm{~mm}$ by 66.7 $\mathrm{mm}$ ) faces on the parting plane for easy part ejection. As shown in Fig. 2, the parts were made with one of the $109.5 \mathrm{~mm}$ by $6.3 \mathrm{~mm}$ faces on the parting plane. This unusual orientation allows full visualization of the mold cavity from the side of the machine where digital-camera placement was possible. Plus, it also extends the machine's clamping capability to parts with larger planform area. By placing one of the smaller edges in contact with the stationary half of the mold, higher in-mold pressures could be reached than would be possible with a more typical orientation of the mold cavity and the parting plane.

A disadvantage of the chosen cavity orientation was part-ejection difficulty caused by frictional contact between the largest faces of the molded part and fixed mold surfaces. This necessitated longer-than-typical cooling times to promote part shrinkage, and, at times, loosening or removal of the large PIV window to allow ejection of the solidified part. Another restriction resulting from this orientation is that the gate must be placed along the cavity face that is in contact with the stationary half of the mold. In this study, the gate was a 3.1-mm-diameter semicircular channel centered $5 \mathrm{~mm}$ above the lower right corner of the part (Figs. 2 and 3).

The mold temperature was monitored with an insulated T-type thermocouple mounted $6 \mathrm{~mm}$ below the surface of the large mold surface on the metal side of the mold. A second thermocouple was used to monitor the ambient air temperature.

\section{Characterizing the Polymer and Particle Mixture}

Molten plastics are non-Newtonian fluids and require detailed characterization. The plastic used in this study, STYRON 615APR, is an FDA compliant all-purpose polystyrene typically used in medical, pharmaceutical, and packaging applications. It is commercially available, relatively easy to mold, and transparent in both the liquid and solid states. STYRON 615APR has a specific gravity of 1.04 and a measured zero-shear viscosity of $295 \pm 3$ $\mathrm{Pa} \mathrm{s}$ at $245^{\circ} \mathrm{C}$. A unique feature of PIV in this study is that the particles and plastic must be mixed in the solid state. Here, the particles and plastic pellets were weighed separately, mixed together by shaking, and simply poured together into the hopper of the injection-molding machine.

Samples of the polymer and particle mixture were tested at $245^{\circ} \mathrm{C}$ by a commercial laboratory where viscosity measurements were performed on a Goettfert Rheograph 2003 capillary rheometer (viscosity error $\pm 2.49 \%$ ) using a representative mixture of STYRON615 APR pellets and PIV particles. Lower shear rate viscosity measurements were made by invoking the Cox-Merz Rule [1] on dynamic test results obtained from a Rheometrics ARES cone and plate rheometer (viscosity error \pm $1.17 \%$ ) using $25-\mathrm{mm}$-diameter disks cut from the center of plaques molded at $230^{\circ} \mathrm{C}$ at an injection rate of 23.0 $\mathrm{cc} / \mathrm{s}$. The resulting viscosity measurements are shown in Fig. 4.

This composite viscosity curve can be fitted using the Carreau model [18].

$$
\eta=\eta_{0}\left[1+(\tau \dot{\gamma})^{2}\right]^{\frac{n-1}{2}}
$$

where $\eta$ is the viscosity, $\eta_{0}$ is the zero-shear viscosity, $\tau$ is a time constant, $\dot{\gamma}$ is the shear rate, and $n$ is the powerlaw exponent. The best-fit Carreau parameters leading to the curve shown in Fig. 4 are $\eta_{0}=295 \pm 3 \mathrm{~Pa} \mathrm{~s}, \tau=$ $0.019 \pm 0.001 \mathrm{~s}$, and $n=0.42 \pm 0.01$. The shear thinning behavior shown in Fig. 4 is common for molten plastics. At high-strain rates, the Carreau model reduces to the simpler power law model.

$$
\eta=m \dot{\gamma}^{n-1}
$$

with $n=0.42$ and $m=\eta_{0} \tau^{n-1}=2900 \pm 100 \mathrm{~Pa} \cdot \mathrm{s}^{n}$.

The time constant from the Carreau model can be used to estimate the Deborah number $D e$ [18], defined as the ratio of the characteristic time of the fluid to the characteristic time of the flow. Solid-like behavior is observed for $D e \gg 1$ and fluid-like behavior is observed for $D e \ll 1$. Viscoelastic behavior is observed for $D e \sim \mathrm{O}(1)$. Here, $D e$ can be evaluated as the $\tau$ determined from the viscosity fit divided by mold-cavity filling time to find $D e$ $\sim 0.005$ to 0.01 ; thus, viscoelasticity should play little or no role in the flow studied in this work. 


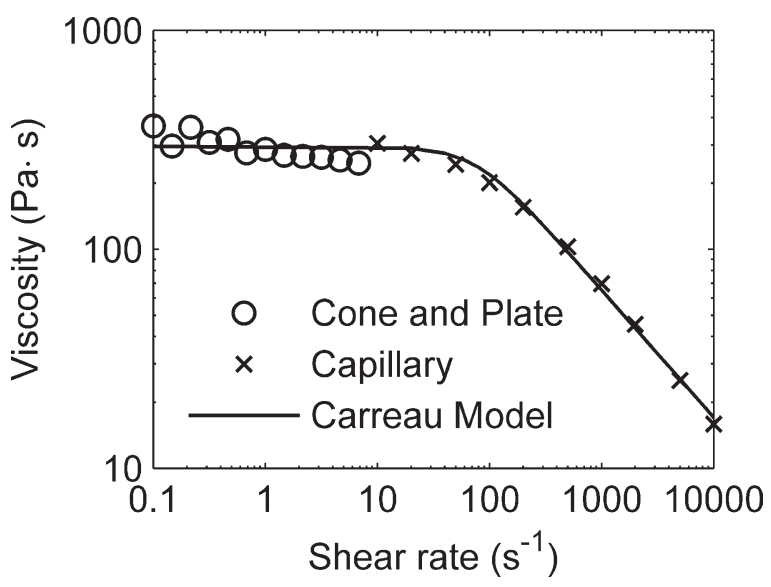

FIG. 4. Viscosity data for STYRON 615APR with $0.02 \%$ (by weight) loading of aluminum flakes at a temperature of $245^{\circ} \mathrm{C}$. The circles represent the magnitude of the complex viscosities determined by an ARES cone and plate rheometer (viscosity error $\pm 1.17 \%$ ). The squares represent data taken with a Goettfert Rheograph 2003 capillary rheometer (viscosity error $\pm 2.49 \%$ ). The Cox-Merz rule was used to combine the two data sets. The solid line represents a best fit of the Carreau model with constants $\eta_{0}=295 \pm 3 \mathrm{~Pa} \mathrm{~s}, \tau=0.019 \pm 0.001 \mathrm{~s}$, and $n=0.42$ \pm 0.01 .

The polymer-particle mixture was also tested by Moldflow Plastics Labs using an injection molding rheometer over a temperature range of $220.1-273.4^{\circ} \mathrm{C}$. The CrossWLF [19] coefficients were calculated from this data and are presented in Table 1.

In addition, the first normal stress coefficient $\Psi_{1}[18]$ was measured as a function of shear rate $\dot{\gamma}$ for the polymer-particle mixture and is shown in Fig. 5. The first normal stress coefficient is zero for Newtonian fluids and indicates that normal stresses are isotropic. It is reported here to complete the characterization of the molten plastic.

\section{PIV Particle Selection}

There are many practical issues in particle selection for molten plastic PIV that are not found in standard PIV experiments. Most of these issues stem from the fact that the PIV particles must be introduced into the plastic's flow path, whereas in the plastic is still solid pellets. Many of the particles typically used in PIV experiments do not survive the plastic melting process in the barrel of the injection-molding machine.

During the course of this experiment, many different particles having a nominal size of several tens of microns were considered and tested. These included copper

TABLE 1. Cross-WLF parameters for STYRON615 APR with $0.02 \%$ by weight loading of aluminum flakes.

\begin{tabular}{ccccccc}
\hline$n$ & $\tau(\mathrm{Pa})$ & $D 1(\mathrm{~Pa} \mathrm{~s})$ & $D 2(\mathrm{~K})$ & $D 3(\mathrm{~K} / \mathrm{Pa})$ & $A 1$ & $A 2(\mathrm{~K})$ \\
\hline 0.3720 & 21765.5 & $4.32561 \mathrm{e} 10$ & 373.15 & 0 & 25.245 & 51.600 \\
\hline
\end{tabular}

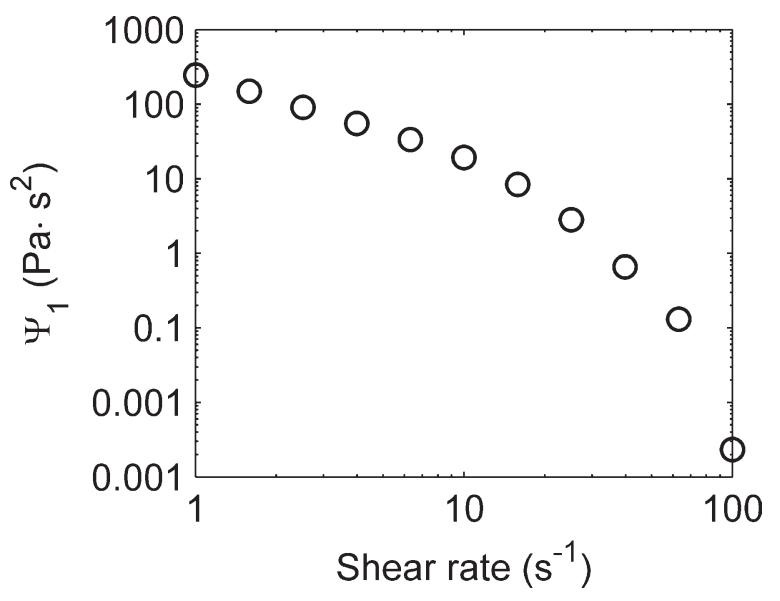

FIG. 5. First normal stress coefficient $\Psi_{1}$ for STYRON 615APR with $0.02 \%$ (by weight) loading of aluminum flakes at a temperature of $245^{\circ} \mathrm{C}$, (error $\pm 1.17 \%$ ).

spheres, aluminum spheres, aluminum oxide powder, uncoated aluminum powder, titanium dioxide powder, hollow glass spheres, silvered glass spheres, fluorescent plastic spheres, silvered plastic flakes, and square-cut aluminum glitter. The particle that was finally selected is manufactured under the trade name Alu*flake by the Glitterex Corporation. These clear-coated precision-cut square aluminum flakes measuring $0.1 \mathrm{~mm} \pm 0.03 \mathrm{~mm}$ on a side with a thickness of $0.013 \mathrm{~mm}$ were tough, highly reflective, and had an equivalent spherical diameter of $60 \mu \mathrm{m}$.

To measure the impact of the particles on the molten plastic viscosity, several tests were conducted using an Instron 1611 capillary rheometer. Viscosity curves were measured using virgin STYRON 615APR pellets with and without $0.02 \%$-by-weight aluminum particles at $230^{\circ} \mathrm{C}$ using a capillary with $25.49 \pm 0.03 \mathrm{~mm}$ length and 1.273 $\mathrm{mm} \pm 0.5 \%$ diameter. No discernable difference in the viscosity curves was observed.

\section{Illumination and Image Acquisition}

The PIV system implemented in this study did not involve any special or unique elements. Particle illumination was provided by the beam from a pulsed SpectraPhysics GCR-130-030 Nd:YAG laser that was spread into a 1-mm-thick sheet centered on the mid plane of the mold cavity parallel to its largest faces. The sheet-producing optics was held by a floor-mounted optical rail that was mechanically isolated from the injection-molding machine to prevent vibration transmission to the optical train. The laser's pulses carried an energy of $93 \mathrm{~mJ}$ and were nominally 6-7 ns in duration.

Planar images of the particles on the center plane of the mold were acquired with a Pulnix TM-9701 camera having a resolution of 600 by 480 pixels running at 30 frames per second. The vertical sync signal was stripped from the camera and used to trigger the pulsed laser. During an experiment, the laboratory was darkened and the 
camera was run with an open shutter since the laser-pulse exposure time was small enough to freeze the moving particles at the $10-20 \mathrm{~cm} / \mathrm{s}$ flow speeds encountered in these experiments. The acquired images were transmitted digitally to a PC computer using Video Savant 3.0 software where they were stored for subsequent analysis.

The overall imaging system was geometrically calibrated to remove radial and tangential optical distortion using a nonlinear image correction scheme [20]. A checkerboard target was placed in the mold at various orientations. The mold was closed and the CCD camera was used to take calibration images of the checkerboard target. All mold glass was in place during this procedure. During postprocessing of the calibration images, the corners of the checkerboard squares were located and the target orientation was calculated. A sixth-order distortion model was then created, and the radial and tangential distortions of the calibration images were calculated. This model was then used to correct the distortions in the movies of the polymer melt flow. In the current experiments, mold filling typically took $1-4 \mathrm{~s}$ and was captured on 30 to 120 frames. Each frame was corrected using this procedure before PIV analysis. The standard deviation of the image correction across an entire image frame was typically 1.0 pixels.

\section{Considerations for Use of PIV in Molten Plastics}

Injection molding involves the unsteady, nonisothermal flow of a shear thinning, viscoelastic liquid at elevated temperatures and pressures. In this study, the nominal melt pressures were as high as $70 \mathrm{MPa}$ in the barrel of the injection-molding machine and as high as $20 \mathrm{MPa}$ in the mold cavity after it is filled. Plastic temperatures ranged from $20^{\circ} \mathrm{C}$ in the machine's hopper to $245^{\circ} \mathrm{C}$ at the end of the barrel. In the mold cavity, the moving molten plastic has a free surface and it solidifies as it flows. Interestingly, these experimental conditions provide both benefits and challenges to the implementation of planar DPIV in molten plastics, and these considerations are described in this section.

First of all, molten plastics are orders of magnitude more viscous than the Newtonian fluids where PIV is commonly used. This is typically advantageous for the success of PIV. A molten plastic flow tends to have relatively weak spatial and temporal velocity gradients when compared with an equivalent speed water flow; this is especially true when the water flow is turbulent. In this study, the highest Reynolds number based on mold cavity thickness and the zero-shear viscosity is $1.4 \times 10^{-4}$, so the spatial and temporal resolution issues faced in higher-Reynolds-number Newtonian PIV are avoided. In addition, the high viscosity of molten plastics allows denser-than-plastic particles to be used without sacrificing flow-tracking accuracy. However, inducing motion of a high-viscosity fluid requires a large pressure difference which leads to optical-access structuralengineering challenges.
A second beneficial feature for implementing PIV in molten plastic flow involves the shear thinning characteristics of the fluid that blunt the flow profile when compared with an equivalent Newtonian flow. For example, the velocity profile, $u(y)$, for pressure-gradient-driven flow of a power-law fluid in a parallel-wall channel of width $2 B$ is given by the following:

$$
u=u_{\max }\left(1-\left|\frac{y}{B}\right|^{\frac{n+1}{n}}\right)
$$

where $y$ is measured from the center of the channel. This velocity profile is plotted in Fig. 6 for constant $u_{\max }$ and power law indices 0.42 (the value for polystyrene) and 1.0 (a Newtonian fluid). The Carreau model profile is not self similar and is a function of the pressure gradient, which varies throughout the mold cavity. The Carreau profile would lie within the envelope defined by the Newtonian and power-law profiles. In this study, the mid-cavity PIV illumination plane nominally lies at $y=0$ in Fig. 6 , so the PIV-measured velocity is presumed to be $u_{\max }$.

The broad, flat maximum in the power-law-fluid's velocity profile is a boon for the current mid-cavity implementation of PIV because, for a fixed error level, it allows the use of a thicker laser sheet and more misalignment of the laser sheet with respect to the channel center plane $(y=0)$ than that allowed by the parabolic Newtonian-fluid profile.

The third important consideration for the use of PIV to determine mold-cavity center-plane velocities involves the 3D fountain flow that forms near the moving plastic-air interface or flow front. Figure 7 shows schematic streamlines of a cross section of this fountain flow in a parallelwall channel. The frame of reference for these streamlines moves at the speed of the flow front, and stagnation point at the tip of the flow front lies at the center of the channel. Here, the molten plastic flows from the gate toward the flow front in the center of the channel. As the center-

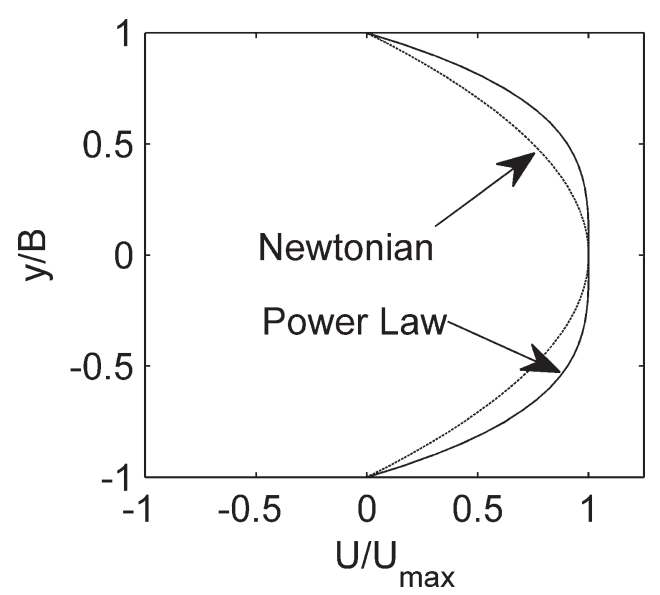

FIG. 6. Fully developed nondimensionalized velocity profiles for a shear-thinning liquid with power law exponent $n=0.42$ and a Newtonian liquid in a channel of width $2 B$. 


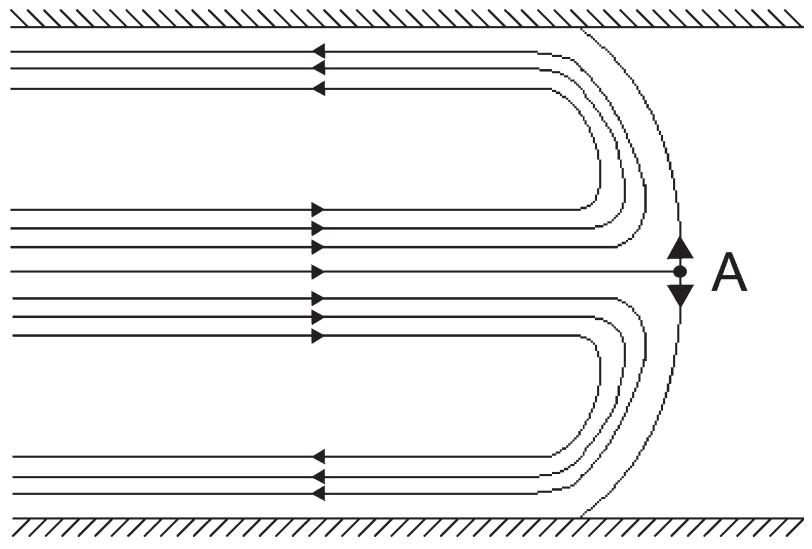

FIG. 7. Schematic of streamlines for fountain flow in a frame of reference traveling with the stagnation point "A." The flow in the center of the channel reaches a stagnation point at the front and is redirected to the walls, causing out-of-plane motion near the front.

plane flow reaches the front, it is divided and redirected, like a fountain, toward the cavity walls where it turns and flows backward (in this frame of reference). In a nonmoving frame of reference, the molten plastic does not flow backward. The plastic begins solidification once it has made contact with the wall.

Fountain flow effects the current implementation of PIV in the neighborhood of the flow front because it includes significant noncavity-center-plane velocities and velocity gradients. Therefore, the current PIV results very close to the flow front cannot be readily extrapolated to the mold-cavity walls. In addition, current near-flow-front PIV velocity measurements should not be expected to satisfy the $2 \mathrm{D}$ center-plane continuity equation because of the existence of nontrivial center-plane-normal fountainflow velocity gradients. Fountain flow causes other problems as well. The curvature of the flow front acts as a lens and can cause glare and distort particle image

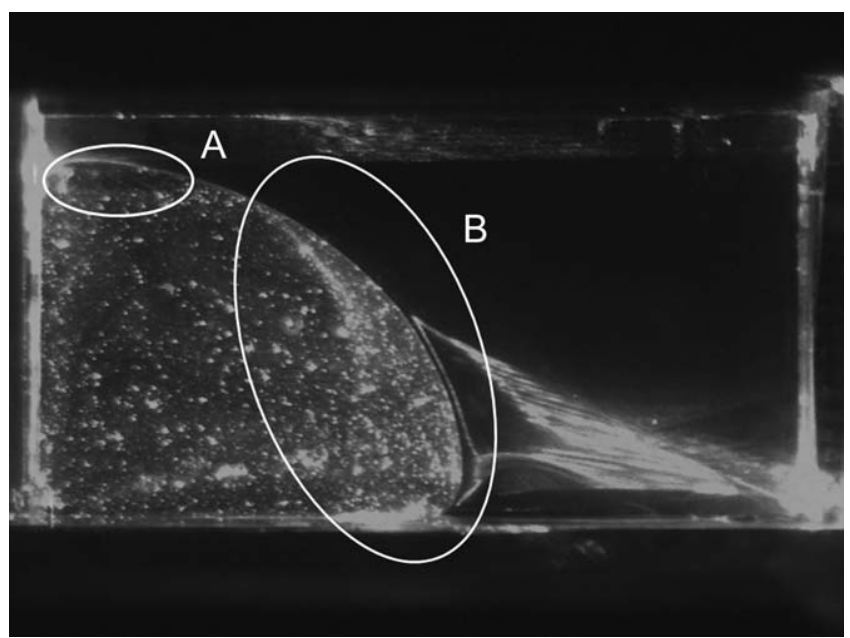

FIG. 8. Uneven illumination due to total internal reflection. In the portion of the image labeled $\mathrm{A}$, the laser sheet is reflected from the interface and fails to illuminate particles in the polymer melt. In the portion of the image labeled B, the laser sheet is reflected back into the polymer melt causing the region behind the front to be overilluminated.

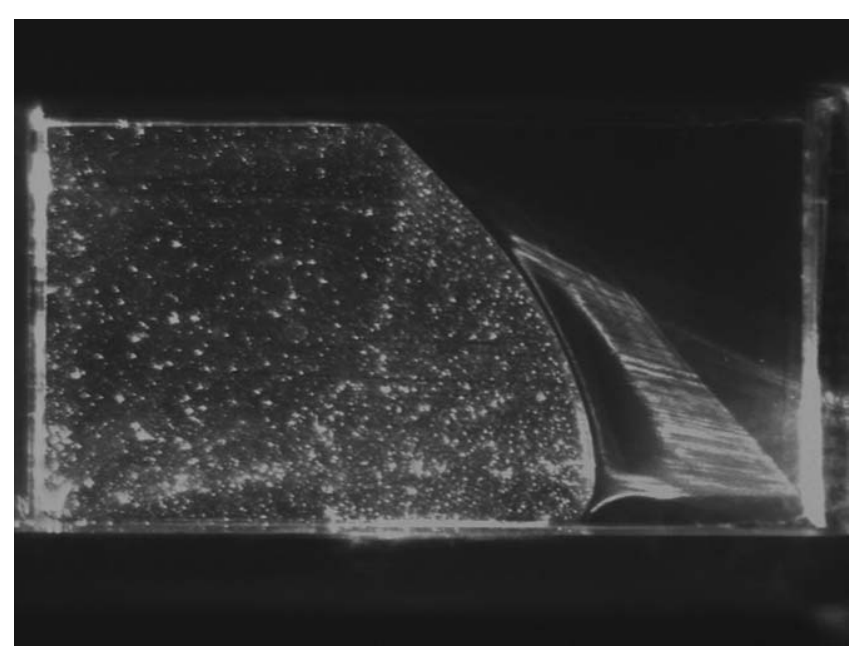

FIG. 9. Uneven illumination due to total internal reflection. In this portion of the mold filling, the front changes shape rapidly and the reflected laser light sweeps through the area behind the front like a searchlight.

motions near the flow front. Fortunately, these two problems, out-of-plane motion and glare, are largely confined to the same regions of the PIV images.

However, laser sheet reflection from, and distortion by, the moving flow front also causes shadows and uneven illumination as the front moves and changes shape during mold filling. Figure 8 illustrates both problems. In this figure, the laser sheet enters the mold cavity on the left, the gate is near the lower left corner of the image, the speckled semicircle is the flowing plastic, the speckles are the aluminum glitter, the edge of the semicircle is the flow front, and reflections of varying strength roughly outline the $109.5 \mathrm{~mm}$ by $66.7 \mathrm{~mm}$ dimensions of the mold cavity. Here, the flowing plastic is predominantly moving away from the lower left corner of the image. The bright triangular region ahead of the flow front to the right is an unintentionally illuminated precursor vapor that enters the mold ahead of the molten plastic. At the left edge of the region labeled $\mathrm{A}$, the curvature of the flow front prevents the penetration of the laser sheet creating a shadow zone where the particles are underilluminated. In the region labeled B, the curved flow front reflects the laser light back into the polymer melt, creating a saturated zone in which the particles are overilluminated. These optical effects lead to a tedious balancing of the particle seeding density and laser illumination. If the seed density or laser illumination is too high, this region oversaturates and becomes unusable for PIV image processing. Yet, if the seed density or laser illumination is low enough to completely avoid such oversaturation, other portions of the flow field may be underilluminated.

When the molten plastic reaches the mold-cavity wall nearest to the letter $\mathrm{A}$, the shadow zone issue disappears, but the melt-front interface reflection problem intensifies because the flow front changes shape rapidly and the reflected laser light sweeps further into the region behind the front, see Fig. 9. Thus, the brightness of individual 
particles in the swept region may change drastically from frame to frame, causing problems for the PIV imagecross-correlation software.

In addition, the current implementation of DPIV lacked the frame rate and resolution to measure the center-plane flow near the gate where it is predominantly radial and the largest center-plane velocity gradients exist. One of the chief sources of error in PIV analysis is the presence of velocity gradients within an interrogation volume that cause particles within the same interrogation window to separate between image frames $[21,22]$. When this separation exceeds a particle diameter, the PIV spatial correlation may fail. To estimate the severity of this problem near the gate in this study, consider two particles of diameter $d_{\mathrm{p}}$ that are separated by a distance of $N / 2 M$ in a radial flow of strength $Q$ issuing from a $90^{\circ}$ corner in a channel of width $2 B$, where $N / 2$ is the half width of the interrogation window in pixels and $M$ is the image magnification factor in units of pixels/unit length. After a time $\Delta t$, the particles will separate by a particle diameter if they are within a critical radius $R_{\mathrm{c}}$ of the flow source, where

$$
R_{\mathrm{c}}=\sqrt{\frac{Q N \Delta t}{8 \pi M d_{\mathrm{p}} B}}
$$

For this work, the maximum flow rate was $Q=34.5 \mathrm{cc} / \mathrm{s}$, the particle's equivalent diameter is $60 \mu \mathrm{m}, \Delta t$ is $1 / 30 \mathrm{~s}$, the channel width $2 B=6.3 \mathrm{~mm}$, and the magnification factor $M$ is 5.2 pixels $/ \mathrm{mm}$, giving a maximum critical radius of $38.6 \mathrm{~mm}$. Thus, standard PIV techniques may be unreliable or fail altogether for particles whose radial distance from the gate is smaller than $R_{\mathrm{c}}$. So, when the sample PIV results are displayed, a semicircular region of radius $R_{\mathrm{c}}$ near the gate is excluded.

An issue that went largely unaddressed in this study is the temperature dependence of the plastic's index of refraction and the nonisothermal character of the flow; it is cooler at the mold's wall than at the mold center plane. The optical effect of nonisothermal flow leads to potential variation of the laser sheet thickness within the mold cavity. The molten plastic's index of refraction decreases with increasing temperature [23] so the net effect of the nonisothermal flow is a slight defocusing of the laser sheet.

Figure 9 may be used to estimate the index of refraction of the polystyrene melt. The laser sheet enters from the left with rays parallel to the long edges of the mold. Parallel streaks of light are also visible in the precursor gas leading the flow front, a gas that is given off during the preparation of the melt in the barrel of the injectionmolding machine. These parallel streaks of light can be extended back to the flow front and the angles of incidence and refraction with the melt front can be measured. Snell's Law can then be used to calculate the index of refraction of the polymer melt, leading to a value of
$1.45 \pm 0.02$. The nominal index of refraction of solid STYRON615APR as provided by the manufacturer is 1.59 .

As mentioned earlier, the relatively high viscosity of the molten plastic allowed the use of metallic particles in this study. The standard requirement for a particle to track the flow is for the Stokes number, $S t=d_{\mathrm{p}}^{2} \rho_{\mathrm{p}} / 18 \eta T$, to be much less than unity. Here, $d_{\mathrm{p}}$ is the particle diameter, $\rho_{\mathrm{p}}$ is the particle material density, $\eta$ is the viscosity of the fluid, and $T$ is the time it takes to fill the mold cavity. The Stokes numbers of the particles used in these studies ranged from $\mathrm{O}\left(10^{-2}\right)$ to $\mathrm{O}\left(10^{-1}\right)$.

Another tracking issue to consider, especially with heavy particles, is the effect of gravity on particle motion. The slip velocity of a spherical PIV particle subject to a gravitational acceleration $g$ is given by $V_{\text {slip }}=d_{\mathrm{p}}^{2}\left(\rho_{\mathrm{p}}-\rho_{\mathrm{f}}\right) g / 18 \eta$, where $\rho_{\mathrm{f}}$ is the fluid density [24]. For the aluminum flakes and STYRON615 APR used in these experiments, the slip velocity is on the order of $10^{-8} \mathrm{~m} / \mathrm{s}$ and is completely negligible.

These Stokes number and slip velocity calculations are based on the assumption that the particle experiences Stokes drag in a Newtonian fluid. In molten plastics, these assumptions may not apply. There is an extensive literature on the drag of spheres in creeping non-Newtonian fluids [25], but experimental data relating to the nonspherical particle dynamics in non-Newtonian fluids are more rare. Thus, an equivalent spherical diameter must be chosen in order to use the available sphere-drag literature to assess the impact of non-Newtonian fluid parameters on PIV analysis.

The general consensus of available research is that shear-thinning effects enhance the drag on spherical particles [25]. This is good for PIV in polymer melts because it further decreases the Stokes number. Computational studies of the drag coefficient for spheres in Carreau fluids [26] have been compared with experimental results [27]. A functional form was given for $X$, the ratio of the measured drag in a shear-thinning fluid to the Stokes drag in a Newtonian fluid:

$$
X=\left[1+\left(k \frac{2 \tau V_{\text {slip }}}{d_{\mathrm{p}}}\right)^{2}\right]^{(n-1) / 2}
$$

where $k$ is a fit parameter. Setting $k=0.275$ matched a wide range of experimental data within $\pm 10 \%$ [26]. Using a slip velocity of $2.5 \times 10^{-8} \mathrm{~m} / \mathrm{s}$, the $60 \mu \mathrm{m}$ nominal aluminum glitter size, and the Carreau-fit parameters for STYRON615 APR leads to a value of $X=0.99$. Thus, the drag experienced by a particle in the molten polystyrene should be the same as that experienced by a particle in a Newtonian fluid with a viscosity equal to the zeroshear viscosity of the molten polystyrene. This result is in accord with the general consensus that shear thinning effects are neutral or somewhat enhance the Stokes drag [25]. 
Viscoelasticity can also affect particle drag. Viscoelasticity typically reduces particle drag [25] but its effects are completely overshadowed by shear-thinning effects at the low Reynolds numbers of this study.

The final particle-motion consideration involves particle migration across shear planes in non-Newtonian flow. In a series of tubular Couette and Poiseuille flows of shear thinning and viscoelastic fluids, Gauthier et al. [28, 29] demonstrated that neutrally buoyant rigid spherical particles moved laterally, a phenomenon that would not be observed in Newtonian flows. For shear thinning fluids, particles tended to move in the direction of the shear stress gradient, from the center toward the outer wall. The migration velocity dropped with increasing distance from the tube axis. For viscoelastic fluids, the behavior was reversed. Particles moved in the direction of decreasing shear stress gradient, from the walls toward the tube axis. The migration velocity dropped with decreasing distance from the tube axis. In both cases, the migration velocities increased with increasing flow rate.

This phenomenon raises concerns for PIV in molten plastics. Such particle migration can lead to out-of-plane motions [21] that cause particles to enter or leave the laser sheet between consecutive PIV images. It is difficult to gauge the magnitude of this effect in injection-molding flow due to a lack of information in the literature. Gauthier's experiments on cylindrical flows have not been extended to channel flows, the more typical injectionmolding geometry. However, since the shear stress gradient approaches zero at the center plane of an injectionmolding flow, any particle migration effects there should be small.

Particle migration due to viscoelasticity must also be considered. The lateral migration rate of a rigid sphere in an unbounded second-order fluid has been calculated, assuming low particle Reynolds number and low Weissenberg number $[30,31]$. Applying these results to a pressure-gradient-driven channel flow gives the following relation for the lateral particle velocity [32]:

$$
U_{\text {lateral }}=-\frac{482}{25} \frac{N_{1}}{\eta}\left(1+\frac{259}{241} \alpha\right)\left(\frac{a}{2 B}\right)^{2} \frac{q^{2}}{16 B^{4}} y
$$

where $a$ is the radius of the sphere, $B$ is the channel half width, $N_{1}$ is the first normal stress difference, $\alpha$ is the negative of the ratio of the first and second normal stress differences, and $q$ is the 2D volumetric flow rate. For STYRON 615APR, rheometry measurements produced $N_{1}=1000 \mathrm{~Pa}$. A value for the second normal stress difference was estimated as $0.4 \alpha$ [33]. The highest value of the volume flow rate used in this work is $34.5 \mathrm{cc} / \mathrm{s}$ and dividing it by the specimen width of $66.7 \mathrm{~mm}$ gives $Q=$ $5.25 \mathrm{~cm}^{2} / \mathrm{s}$. Taking $y=0.5 \mathrm{~mm}$ to be the edge of the laser sheet and using these values gives a worst-case estimate of the particle migration velocity $U_{\text {lateral }}=-0.17$ $\mathrm{mm} / \mathrm{s}$, the negative sign indicating that the migration is toward the mid plane of the mold cavity. For the conditions described earlier, typical PIV velocities were on the order of 3 to $20 \mathrm{~cm} / \mathrm{s}$, indicating that particle migration due to viscoelastic effects will be negligible. Moreover, this migration velocity approaches zero for particles closer to the mold-cavity mid plane.

\section{Image Analysis}

The raw 480 by 600 camera frames required significant preprocessing before PIV analysis. First, the raw images were corrected to account for geometrical optical distortion as described earlier. The cavity boundaries and the flow front were then manually extracted from the images. The flow fronts were used to calculate the local flow front velocities from differences between images and the assumption that the flow front always moved perpendicular to itself. The flow fronts and cavity boundaries were used to create binary masks to isolate the polymer melt from the rest of the image. The masked images were then used as the inputs for the PIV analysis.

The PIV analysis was accomplished with DaVis 6.2.2 software by cross-correlating square windows within consecutive images from the mold-filling movies. Adaptive multipass was used employing $128^{2}-, 64^{2}$-, and $32^{2}$-pixel ${ }^{2}$ windows with $50 \%$ overlap, resulting in a grid of displacement vectors spaced by 16 pixels $(3.5 \mathrm{~mm})$. The resulting data was smoothed using a $3 \times 3$ Gaussian filter. The PIV output was then scaled to laboratory coordinates and multiplied by the camera frame rate to calculate the velocity vectors.

The lack of control in obtaining an even distribution of particles throughout the flow field and the illumination problems caused by melt-front shadowing and reflections lead to large gaps in any single PIV field where velocity data could not be retrieved. The locations and number of these gaps would vary unpredictably from cycle to cycle. This situation is illustrated in Fig. 10 which shows two PIV fields at the $90 \%$ fill time for two different specimens made under the same molding conditions during the same run of parts. Velocity vector drop outs are evident in both fields, but in different locations.

Fortunately, the overall flow field was repeatable and smooth, even if the particle density was inconsistent. Thus, different PIV movies made under the same conditions were pooled to overcome the random defects in particle density and illumination.

Sets of six to eight PIV vector fields for the same processing conditions were superposed for each time of interest, 30, 50, 70, and $90 \%$ of the fill time. Median velocity components were selected at each grid point. The median was chosen rather than the average at each point since the median is more robust and is less likely to be affected by velocity vector dropout and undetected spurious velocity vectors. The intended effect of taking the median at each spatial location was to eliminate the 


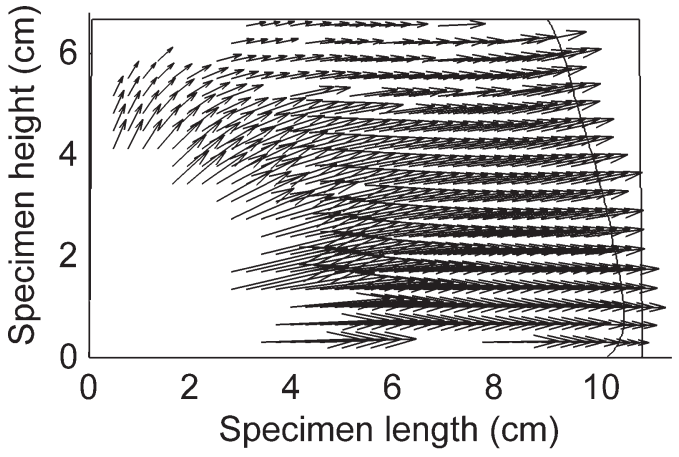

(a)

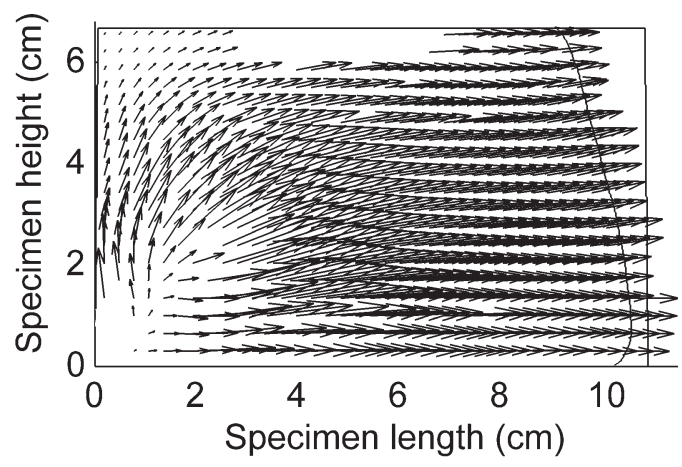

(b)

FIG. 10. PIV vector fields computed at the $90 \%$ fill time for two different specimens molded under the same conditions during the same run of parts. The PIV fields have missing vectors in different places of the flow field. The PIV fields also show the failure of the PIV software to resolve the correct vectors near the gate.

spatial data gaps. An example of the outcome after median filtering is shown in Fig. 11.

To assess shot-to-shot repeatability, the flow fronts from the superposed PIV images were also superposed on each other and the entire set used as an input to a polynomial-fitting scheme. Flow front positions fell within a range of $\pm 1 \mathrm{~mm}$ around the best-fit front. As an example, Fig. 12 shows a superposition of flow fronts from six data sets and the best fit curves calculated from them.

To estimate the error involved in median filtering, the set of velocity vector components were sorted at each point. The two vector components bracketing the median value were examined to determine a range about the median. This range was typically $\pm 2 \%$ of the median value.

The overall accuracy of the median-filtered PIV fields was assessed through a calculation and nondimensionalization of residuals of the continuity equation. The polymer flow in this study is effectively incompressible during the mold-filling process, and the mold-cavity center-plane flow can be considered $2 \mathrm{D}$ except for the region near the front where fountain

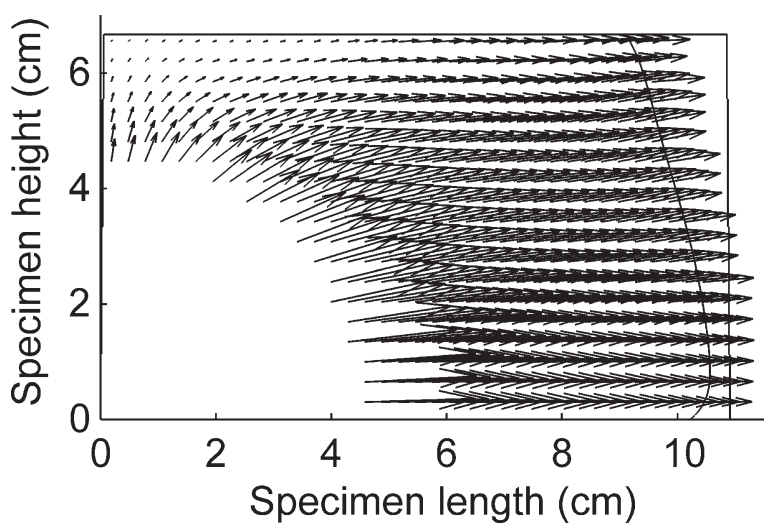

FIG. 11. The result of median filtering a set of seven PIV fields calculated at the same point of fill from specimens molded at identical conditions. Two of the PIV fields used in the set are shown in Fig. 10. A dead-zone radius was applied around the gate to eliminate vectors from the region where the PIV analysis fails. flow occurs. Here, the continuity equation, $\nabla \cdot \vec{v}=0$, was approximated in two dimensions as follows:

$$
\frac{\Delta u}{\Delta x}+\frac{\Delta v}{\Delta y}=\varepsilon(x, y)
$$

where $\Delta u / \Delta x$ and $\Delta v / \Delta y$ are numerical approximations of the velocity gradients based on the central differences of PIV vector components and grid spacings, and $\varepsilon$ is a residual or error term with units of inverse time. This error will be a function of position for any given PIV vector field. The error arises from three-dimensionality in the flow field, inaccuracies in the PIV data itself, and from the inherent numerical error arising from discrete approximations of derivatives.

Since the velocities within an interrogation volume were considered constant, the error term is also constant within an interrogation volume. Integrating the error over the interrogation volume results in a quantity with the units of volume flow rate. This quantity may be scaled by the injection flow

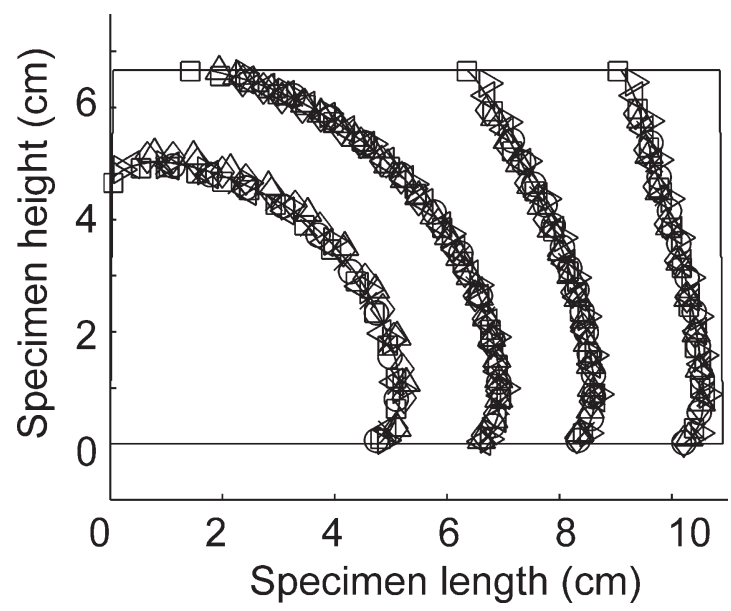

FIG. 12. Flow fronts extracted from data sets molded at identical conditions at $30,50,70$, and $90 \%$ of the fill time. The data points shown are subsets of the actual data for clarity of plotting. The best-fit fronts calculated from these fronts are also displayed. 


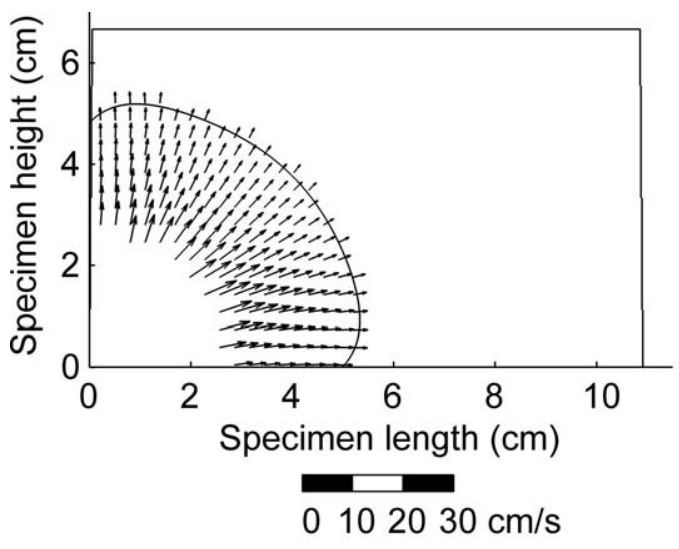

(a)

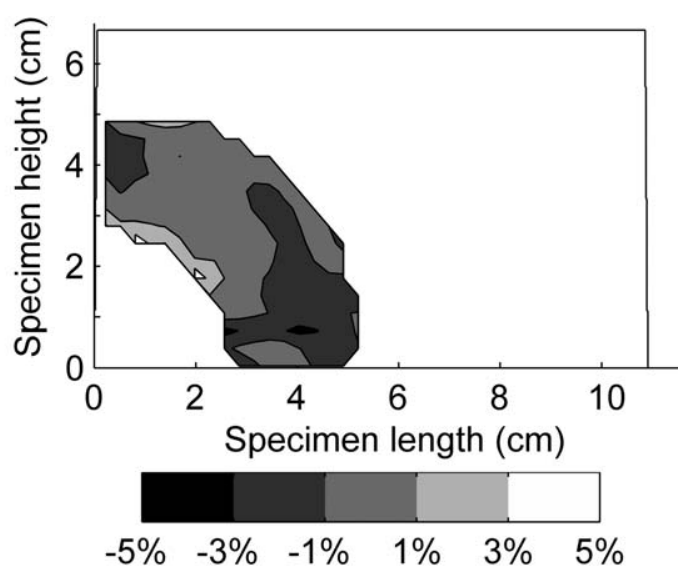

(b)

FIG. 13. PIV vectors (a) and scaled continuity contours (b) for an injection flow of $11.5 \mathrm{cc} / \mathrm{s}$ at $245^{\circ} \mathrm{C}$ at $30 \%$ of the total fill time of $4 \mathrm{~s}$. The region around the gate in the lower left corner cannot be resolved due to particle separation between image frames. The color bar below the contour plot shows the scaled continuity error as a percentage of the injection flow rate. An error of 5 on this scale corresponds to an artificial volume source equal to $5 \%$ of the magnitude of the injection flow rate.

rate of the flow to give a nondimensionalized form of the continuity-equation error as follows:

$$
\varepsilon^{*}=\frac{V_{\mathrm{I}} \varepsilon}{Q}
$$

where $V_{\mathrm{I}}$ is the volume of an interrogation cell and $Q$ is the injection rate of the flow. Here, the dimensionless $\varepsilon^{*}$ can be interpreted as a field of volume sources and sinks scaled by the volume flow rate of the injection flow through the gate. If $\varepsilon^{*}=0$ at a point, then the continuity equation is satisfied perfectly at that point. As the magnitude of $\varepsilon^{*}$ at a point approaches \pm 1 , the erroneous source or sink strength at that point approaches that of the injection flow rate.

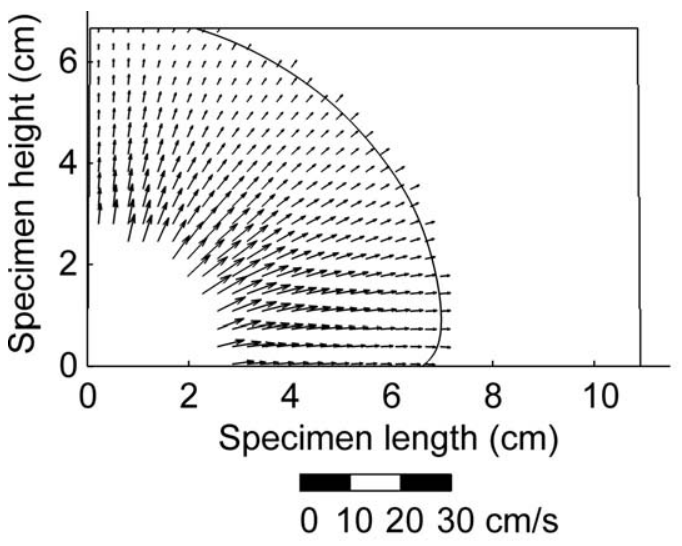

(a)

\section{SAMPLE EXPERIMENTAL RESULTS}

Figures 13-16 provide a sample of the velocity fields and flow fronts measured with the experimental apparatus and techniques described earlier. The molten plastic was at a temperature of $245^{\circ} \mathrm{C}$ and the injection rate was 11.5 $\mathrm{cc} / \mathrm{s}$. The median velocity fields and melt fronts shown in these figures are drawn from eight digital movies. Results are shown at $30,50,70$, and $90 \%$ of the fill time of $4 \mathrm{~s}$.

Corresponding contour plots are also presented showing $\varepsilon^{*}$, the nondimensionalized continuity error. These errors were typically in the range of $\pm 2 \%$ with extreme values reaching $-5 \%$. An interesting feature of these plots is the region of apparent flow sinks near the flow front (especially obvious in Figs. 15b and 16b). This is to be expected because of the fountain flow's center-plane-normal velocity gradient at the flow front.

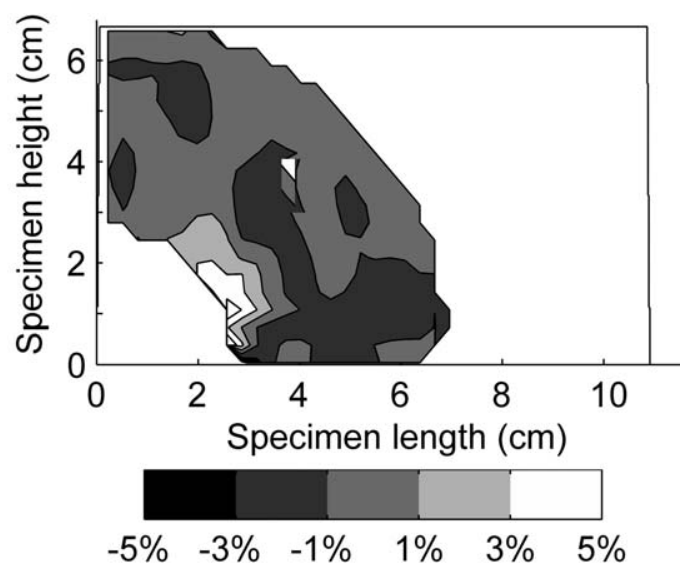

(b)

FIG. 14. PIV vectors (a) and scaled continuity contours (b) for an injection flow of $11.5 \mathrm{cc} / \mathrm{s}$ at $245^{\circ} \mathrm{C}$ at $50 \%$ of the total fill time. 


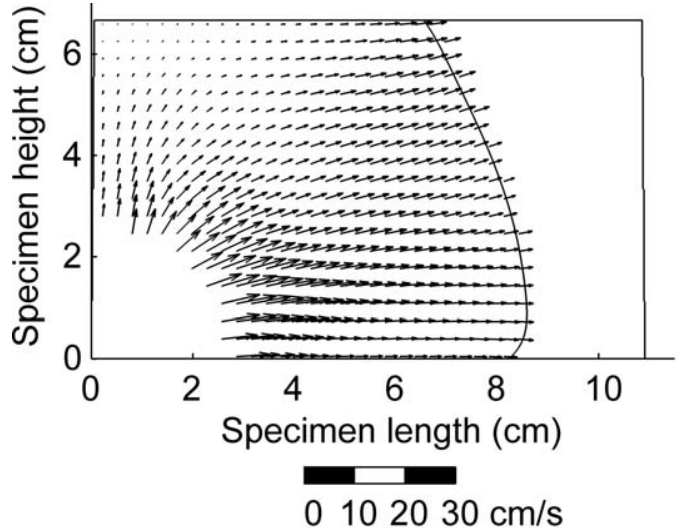

(a)

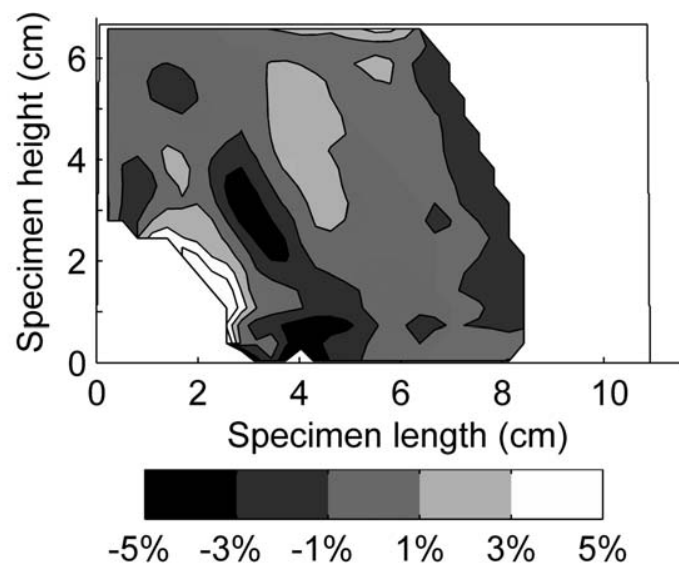

(b)

FIG. 15. PIV vectors (a) and scaled continuity contours (b) for an injection flow of $11.5 \mathrm{cc} / \mathrm{s}$ at $245^{\circ} \mathrm{C}$ at $70 \%$ of the total fill time.

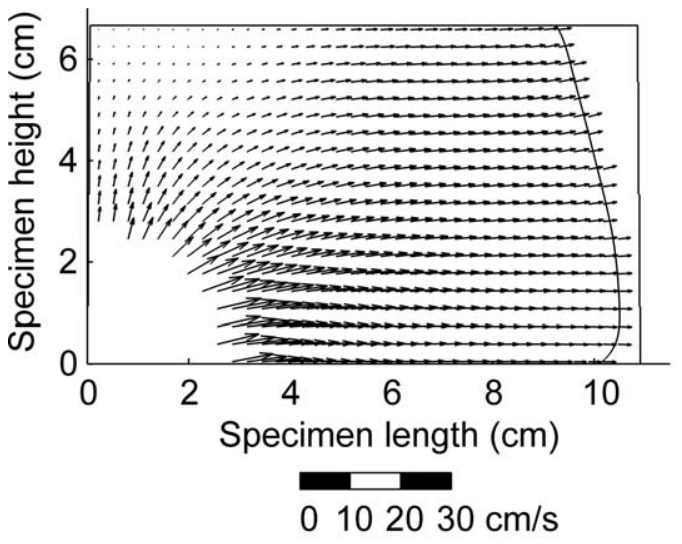

(a)

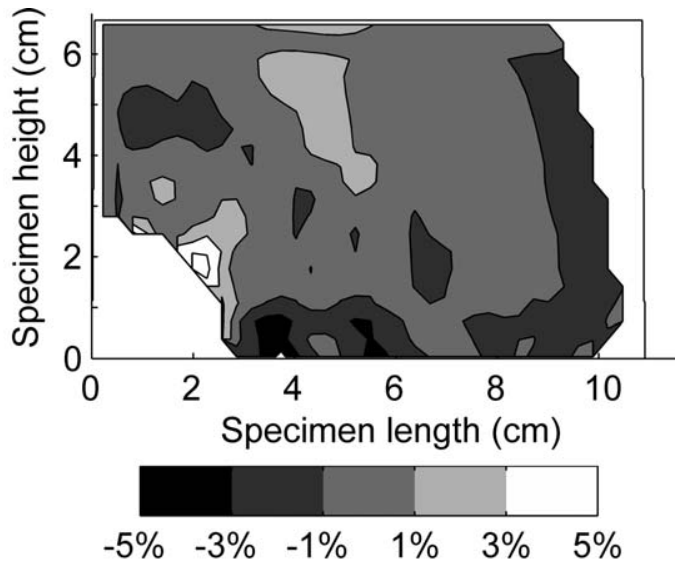

(b)

FIG. 16. PIV vectors (a) and scaled continuity contours (b) for an injection flow of $11.5 \mathrm{cc} / \mathrm{s}$ at $245^{\circ} \mathrm{C}$ at $90 \%$ of the total fill time.

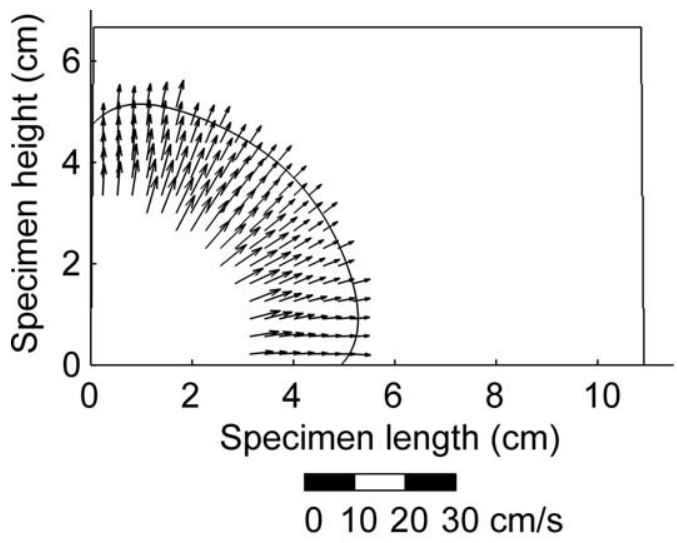

(a)

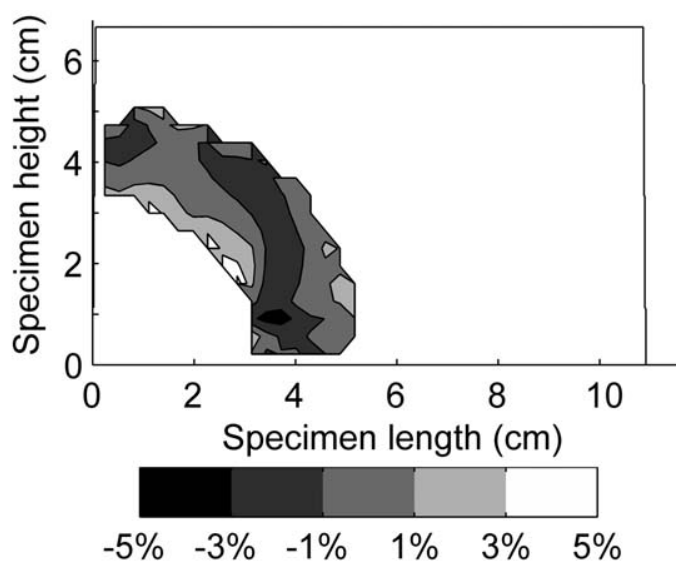

(b)

FIG. 17. PIV vectors (a) and scaled continuity contours (b) for an injection flow of $17.3 \mathrm{cc} / \mathrm{s}$ at $245^{\circ} \mathrm{C}$ at $30 \%$ of the total fill time. 


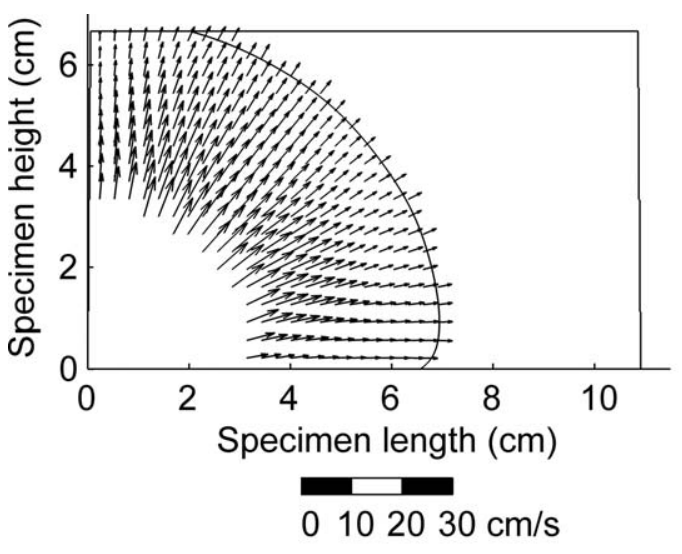

(a)

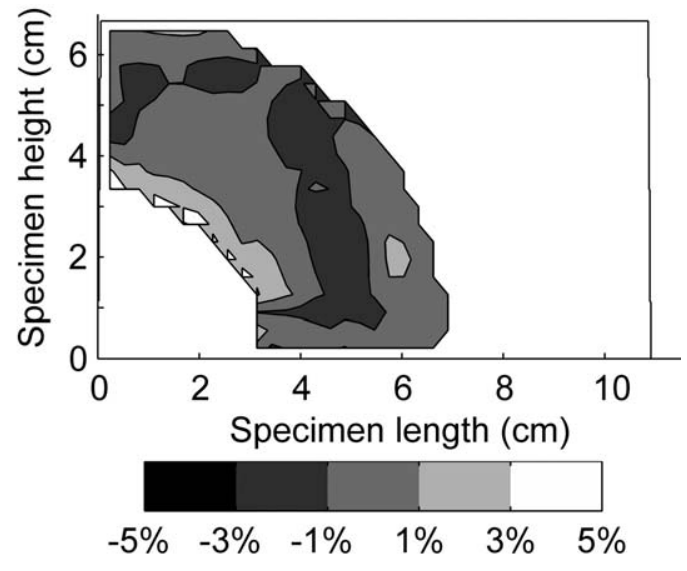

(b)

FIG. 18. PIV vectors (a) and scaled continuity contours (b) for an injection flow of $17.3 \mathrm{cc} / \mathrm{s}$ at $245^{\circ} \mathrm{C}$ at $50 \%$ of the total fill time.

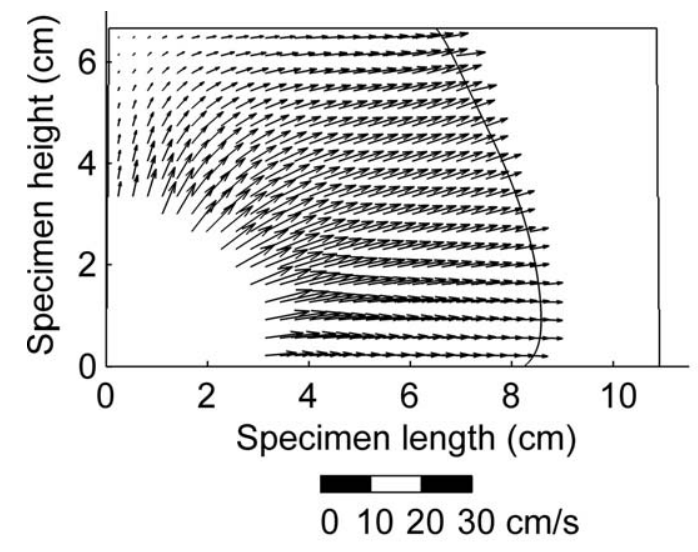

(a)

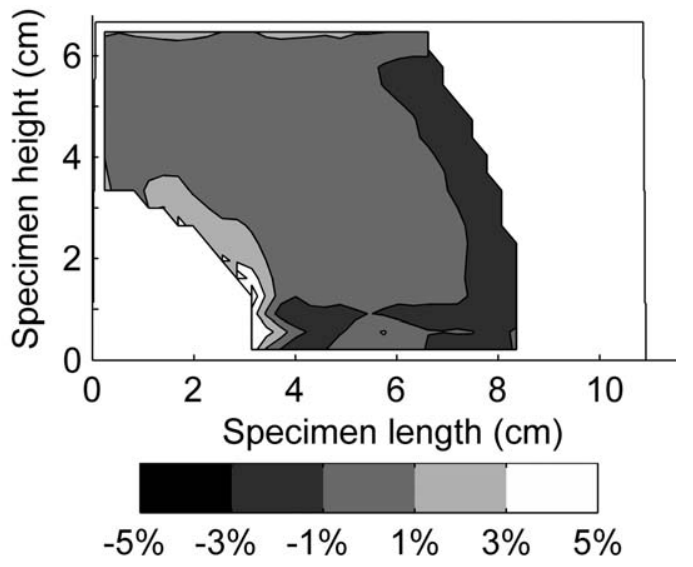

(b)

FIG. 19. PIV vectors (a) and scaled continuity contours (b) for an injection flow of $17.3 \mathrm{cc} / \mathrm{s}$ at $245^{\circ} \mathrm{C}$ at $70 \%$ of the total fill time.

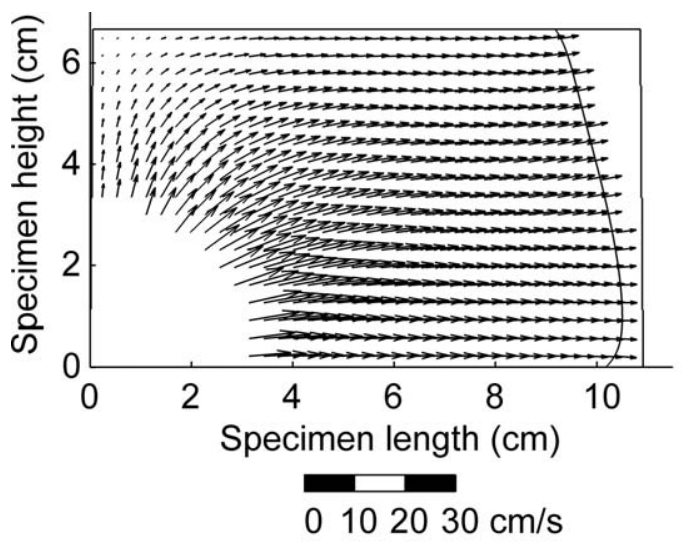

(a)

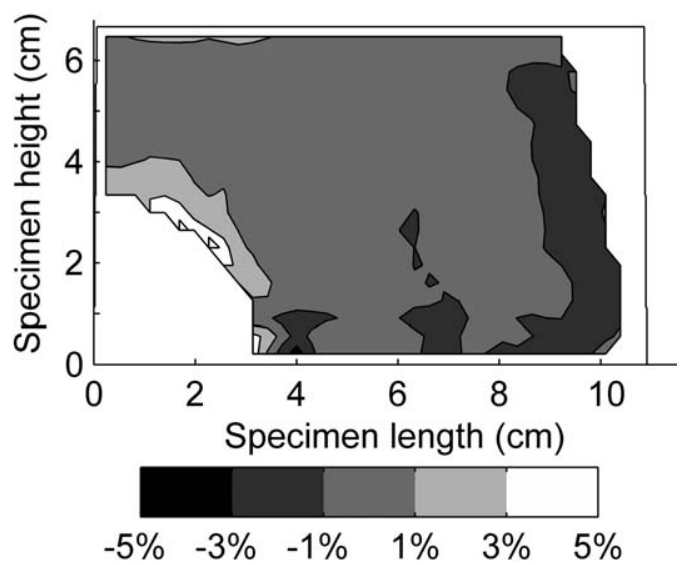

(b)

FIG. 20. PIV vectors (a) and scaled continuity contours (b) for an injection flow of $17.3 \mathrm{cc} / \mathrm{s}$ at $245^{\circ} \mathrm{C}$ at $90 \%$ of the total fill time. 


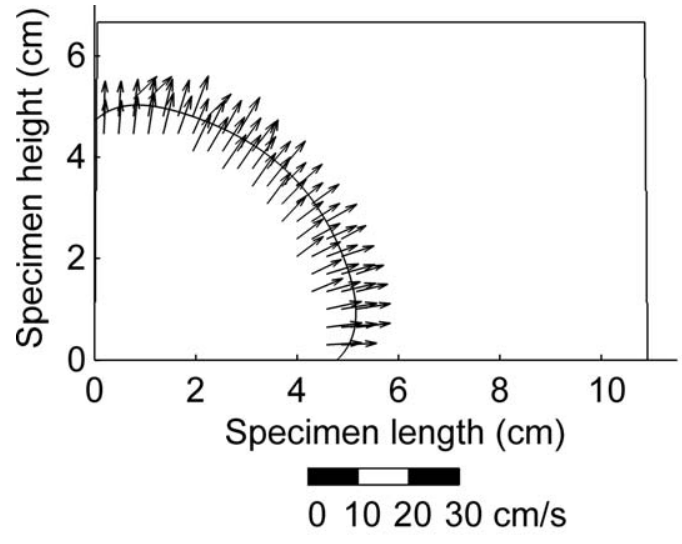

(a)

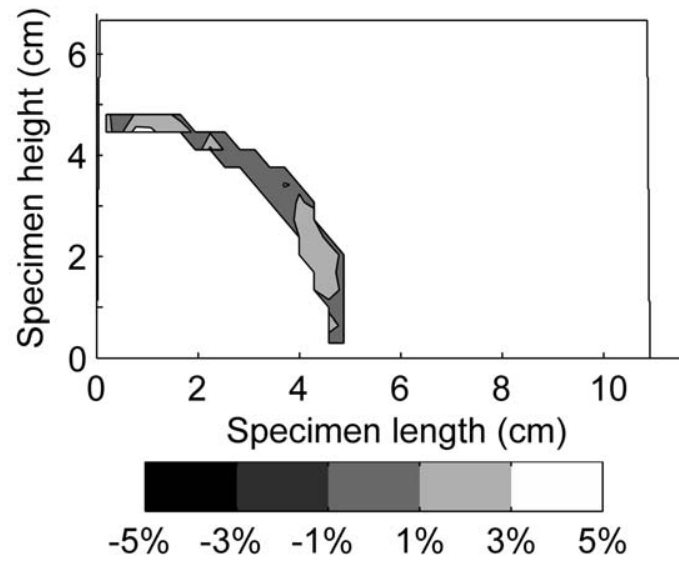

(b)

FIG. 21. PIV vectors (a) and scaled continuity contours (b) for an injection flow of $34.5 \mathrm{cc} / \mathrm{s}$ at $245^{\circ} \mathrm{C}$ at $30 \%$ of the total fill time.

Figures 17-20 show a similar sequence of mid-plane velocity fields and continuity error contour plots for molten plastic at $245^{\circ} \mathrm{C}$ and an injection rate of $17.3 \mathrm{cc} / \mathrm{s}$. Figures 21-24 show the mid-plane velocity fields and continuity error contours for molten plastic at $245^{\circ} \mathrm{C}$ and an injection rate of $34.5 \mathrm{cc} / \mathrm{s}$.

As the injection rate increases, the radius of the zone around the gate where PIV fails also increases as predicted in $E q$. 5. The magnitude of the velocity vectors increases with increasing injection rate, but the orientation of the vectors is unaffected by injection rate for this cavity geometry in the range of injection rates studied. Analysis of the velocity data shows that the vector fields scale linearly with injection rate. For example, the injection rate in Figs. 21-24 was three times the injection rate in Figs. 13-16. If the magnitudes of the velocity vectors shown in Figs. 21-24 are divided by a factor of the 3, the resulting velocity fields are statistically indistinguishable from the velocity fields shown in Figs. 13-16.

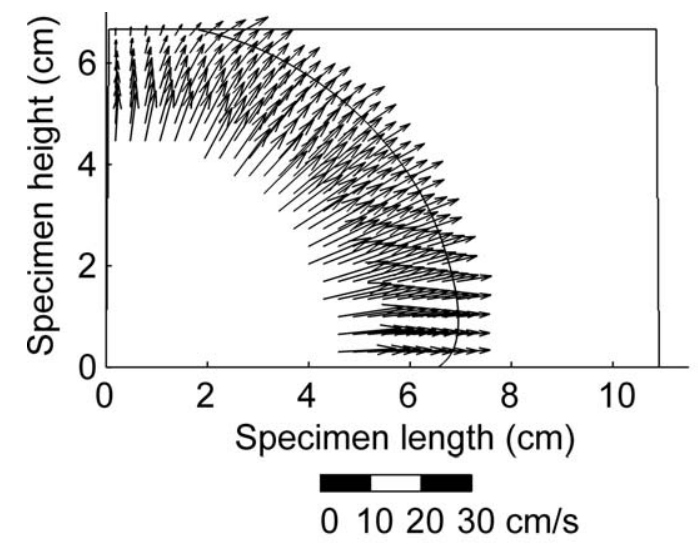

(a)

\section{CONCLUSIONS}

This article describes the experimental techniques and considerations that allow particle image velocimetry (PIV) to be applied to flowing molten polystyrene as it fills the mold cavity in an industrial injection molding machine at temperatures, pressures, and flow rates typical of production processes. In this technique development effort, the experimental challenges included temperatures from $230-245^{\circ} \mathrm{C}$, pressures exceeding $20 \mathrm{MPa}$, and a lack of access to the flowing molten plastic for injecting and mixing seed particles. These challenges were overcome with a customdesigned optical-access mold, aluminum-glitter seed particles that survive the machine's thermal-mechanical plastic melting process, and median filtering of repeated velocity field measurements to obtain complete mold-cavity center-plane velocity fields. In addition, assessments of a variety of shear thinning and viscoelastic effects that could potentially lead to unexpected particle motions suggest that the chosen par-

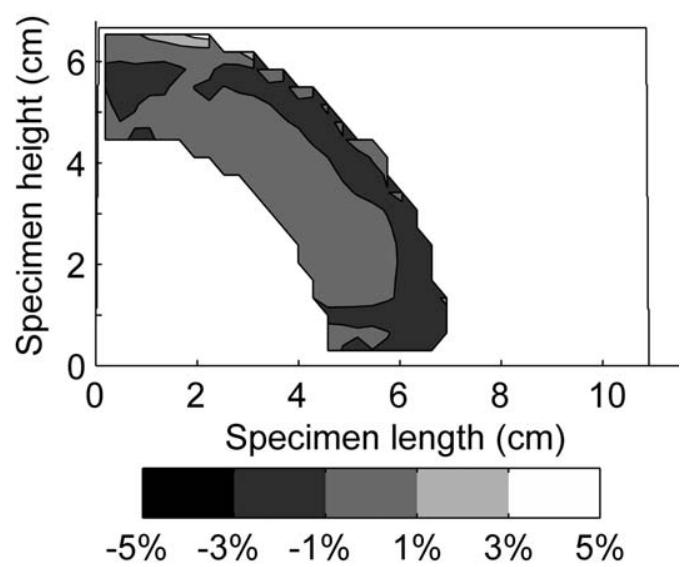

(b)

FIG. 22. PIV vectors (a) and scaled continuity contours (b) for an injection flow of $34.5 \mathrm{cc} / \mathrm{s}$ at $245^{\circ} \mathrm{C}$ at $50 \%$ of the total fill time. 


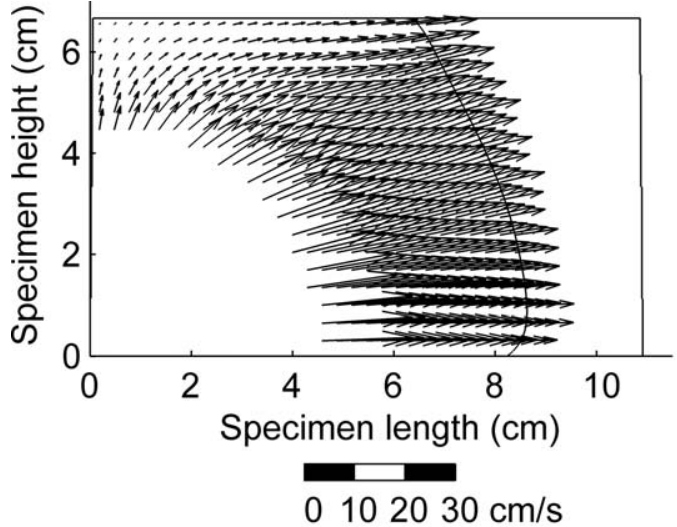

(a)

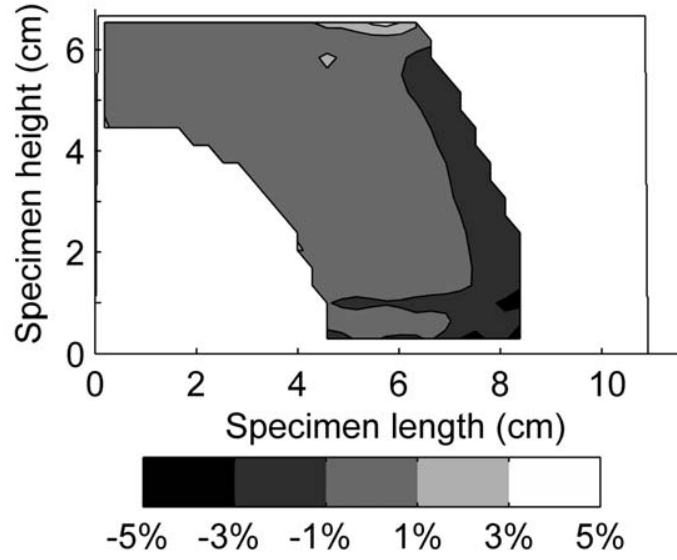

(b)

FIG. 23. PIV vectors (a) and scaled continuity contours (b) for an injection flow of $34.5 \mathrm{cc} / \mathrm{s}$ at $245^{\circ} \mathrm{C}$ at $70 \%$ of the total fill time.

ticles adequately follow the flow of molten plastic. Uncertainty in the final 2D mold-cavity center-plane PIV medianfiltered velocities was determined to be $\pm 2 \%$. Residuals from a discrete 2D evaluation of the continuity equation on the mold-cavity center plane were calculated and found to be typically less than $2 \%$ of the injection flow rate.

The following conclusions can be drawn from this study. (1) The custom-designed optical access mold that included plate glass as a structural element was sufficiently durable for this study with a design safety factor of 10 on the glass plates. Even though the total PIV window thickness was $\sim 10 \mathrm{~cm}$, it provided sufficient optical clarity for PIV measurements. (2) Aluminum glitter was robust and reflective enough for this development effort even though it was merely mixed with the solid plastic pellets before they were melted. The process of injection molding does not readily allow particle mixing after the plastic is melted; thus, adequately reflective particles that survive the plastic melting process are essential. However, lack of control over the particle-mixing process led to seed uniformity problems that necessitated repetition of experiments. (3) The high viscosity and shear thinning character of the molten plastic leads to smoothly varying in-mold flow and a blunted velocity profile between nearby nearly parallel surfaces. Both factors are advantages for the application of PIV in such flows because they allow the use of relatively dense metallic particles and ordinary digital cameras operating at standard video frame rates and because they reduce laser-sheet thickness and alignment concerns. (4) The evolving location and shape of the plastic-air interface (or flow front) within the mold caused particle illumination to be unsteady and this is detrimental for the application of PIV to molten plastic flow during mold filling. When coupled with nonuniform seeding, this problem could only be surmounted by a trial-and-error effort to find an acceptable seeding

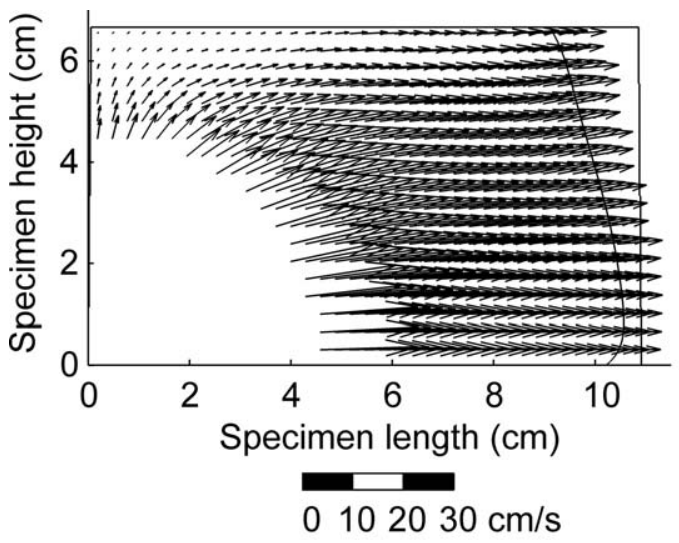

(a)

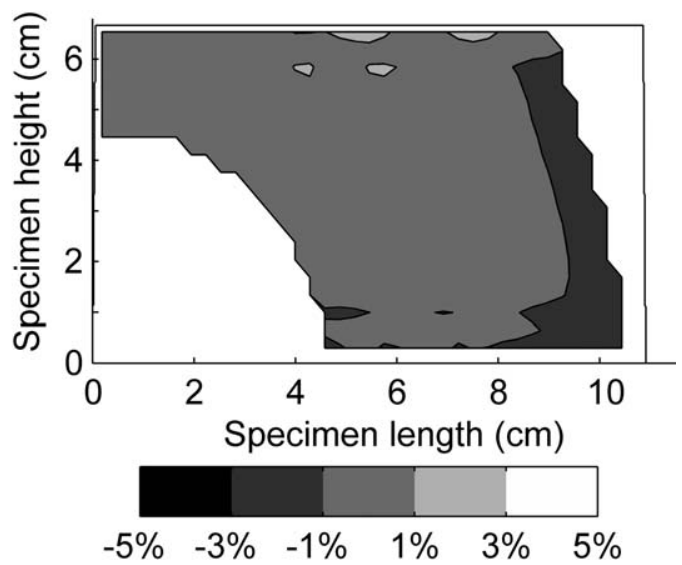

(b)

FIG. 24. PIV vectors (a) and scaled continuity contours (b) for an injection flow of $34.5 \mathrm{cc} / \mathrm{s}$ at $245^{\circ} \mathrm{C}$ at $90 \%$ of the total fill time. 
level and by repeating experiments and combining results. (5) Median filtering is a successful PIV-measurement combination technique for multiple experiments conducted under the same conditions. It is less sensitive to vector drop outs and erroneous vectors than simple averaging, and the nearest-value measurements at any location provide a ready estimate of the median-filtered PIV-field uncertainty. (6) For this cavity geometry, the velocity fields scaled linearly with injection rate for the range of injection rates studied. (7) The $\pm 2 \%$ continuity equation residuals in this study suggest that the techniques described here may be successful enough for quantitative comparisons with mold filling simulations.

\section{ACKNOWLEDGMENTS}

Cincinnati Milacron assisted with the purchase of the injection molding machine.

\section{REFERENCES}

1. J. Dealy and K. Wissbrun, Melt Rheology and Its Role in Plastics Processing, Van Nostrand Reinhold, New York (1990).

2. C. Willert and M. Gharib, Exp. Fluids, 10, 181 (1991).

3. G. Gilmore and S. Spencer, Mod. Plastics, 28, 117 (1951).

4. M. Kamal and S. Kenig, Polym. Eng. Sci., 12, 302 (1972).

5. J. White and H. Dee, Polym. Eng. Sci., 14, 212 (1974).

6. M. Kamal and S. Kenig, Polym. Eng. Sci., 12, 294 (1972).

7. J. White, Polym. Eng. Sci., 15, 44 (1975).

8. S. Fathi and A.H. Behravesh, Polym. Eng. Sci., 47, 750 (2007).

9. S. Fathi and A.H. Behravesh, Polym. Eng. Sci., 48, 598 (2008).

10. L. Tredoux, I. Satoh, and Y. Kurosaki, Polym. Eng. Sci., 40, 2161 (2000).

11. X. Han and H. Yokoi, Polym. Eng. Sci., 46, 1590 (2006).

12. B. Baumert, D. Liepmann, and S. Muller, J. Nonnewton. Fluid Mech., 69, 221 (1997).
13. J. Shoemaker, Moldflow Design Guide: A Resource for Plastic Engineers, Hanser Gardner Publications, Cincinnati (2006).

14. T. Bress and D. Dowling, J. Reinf. Plast. Compos., 17, 1374 (1998).

15. R. Roark and R. Budynas, Roark's Formulas for Stress and Strain, 7th ed., McGraw-Hill, New York (2002).

16. M. Ashby and D. Jones, Engineering Materials 2: An Introduction to Microstructures, Processing and Design, Butterworth-Heineman, Oxford (2006).

17. G. McLellan and E. Shand, Glass Engineering Handbook, 3rd ed., McGraw-Hill, New York (1984).

18. R. Bird, R. Armstrong, and O. Hassager, Dynamics of Polymeric Materials, Vol. 1, Wiley, New York (1987).

19. T. Osswald, L. Turng, and P. Gramann, Injection Molding Handbook, Hanser Gardner Publications, Cincinnati (2008).

20. J. Heikkilä and O. Silvén, IEEE Computer Science Conference on Computer Vision and Pattern Recognition (CVPR'97), San Juan, 1106 (1997).

21. H. Huang, H. Fiedler, and J. Wang, Exp. Fluids, 15, 168 (1993).

22. P. Meunier and T. Leweke, Exp. Fluids, 35, 408 (2003).

23. R. Moshrefzadeh, M. Radcliffe, T. Lee, and S. Mohapatra, J. Lightwave Tech., 10, 420 (1992).

24. M. Raffel, C. Willert, and J. Kompenhans, Particle Image Velocimetry: A Practical Guide, Springer-Verlag, Berlin (1998).

25. R. Chhabra, Bubbles, Drops and Particles in Non-Newtonian Fluids, CRC Press, Boca Raton (1993).

26. M. Bush and N. Phan-Thien, J. Nonnewt. Fluid Mech., 16, 303 (1984).

27. R. Chhabra and P. Uhlherr, Rheol. Acta, 19, 187 (1980).

28. F. Gauthier, H. Goldsmich, and S. Mason, Rheol. Acta, 10, 344 (1971)

29. F. Gauthier, H. Goldsmich, and S. Mason, Trans. Soc. Rheol., 15, 297 (1971).

30. P. Brunn, Rheol. Acta, 15, 589 (1976).

31. P. Chan and L. Leal, J. Fluid Mech., 82, 549 (1977).

32. S. Emerman, Polym. Eng. Sci., 27, 1105 (1987).

33. P. Brunn, J. Nonnewton. Fluid Mech., 7, 271 (1980). 